\title{
Deep Learning Architectures and Techniques for Multi-organ Segmentation
}

\author{
Valentin Ogrean ${ }^{1}$, Alexandru Dorobantiu ${ }^{2}$, Remus Brad ${ }^{3}$ \\ Faculty of Engineering \\ "Lucian Blaga" University \\ Sibiu, Romania
}

\begin{abstract}
Deep learning architectures used for automatic multi-organ segmentation in the medical field have gained increased attention in the last years as the results and achievements outweighed the older techniques. Due to improvements in the computer hardware and the development of specialized network designs, deep learning segmentation presents exciting developments and opportunities also for future research. Therefore, we have compiled a review of the most interesting deep learning architectures applicable to medical multi-organ segmentation. We have summarized over 50 contributions, most of which are more recent than 3 years. The papers were grouped into three categories based on the architecture: "Convolutional Neural Networks" (CNNs), "Fully Convolutional Neural Networks" (FCNs) and hybrid architectures that combine more designs - including "Generative Adversarial Networks" (GANs) or "Recurrent Neural Networks" (RNNs). Afterwards we present the most used multi-organ datasets, and we finalize by making a general discussion of current shortcomings and future potential research paths.
\end{abstract}

Keywords-Deep Learning; Multi-Organ Segmentation; Fully Convolutional Neural Networks (FCNs); Generative Adversarial Networks (GANs); Recurrent Neural Networks (RNNs)

\section{INTRODUCTION}

Medical imaging using Computed Tomography (CT), Magnetic Resonance (MR), ultrasound, X-ray, and so on, has become an essential part in detection, diagnosis, and treatment of diseases [1].

A new medicine branch, imaging and radiology was developed to train human experts that can interpret medical images and provide an accurate diagnosis. The training is challenging due to the complexity involved, but more importantly, the diagnosis process itself is a tedious and exhausting work that is further impacted by the large variations in pathology between different individuals. Therefore, the need for automated help grew larger as the medical imagining sector expanded, with use-cases like segmentation of medical images, delineating human organs or automated diagnosis being intensively studied using Deep Learning (DL) architectures.

Deep learning absorbs the feature engineering designed by human experts into a learning step [2]. Furthermore, deep learning needs only a set of training/testing data with minor pre-processing (if necessary), and then can extract the human body representations in an autonomous manner. Throughout different architectures, DL has demonstrated enormous potential in computer vision [3].
Multi-organ deep learning architectures could lend a helping hand in the field of radiation therapy, by the making the segmentation process faster and more robust [4]. Multiorgan segmentation also paves the way for automation processes that are generalized to the full body or to a large spectrum of diseases facilitating online adaptive radiotherapy and fulfilling medical image segmentation's goal to become autonomous in reaching an accurate diagnosis in any medical imaging environment.

\section{A. Segmentation Applications in the Medical Field}

- Radiotherapy in cancer treatment. In radiotherapy, the need exists to control the radiation exposure of the target and healthy organs, so segmentation of organs at risk (OARs) could provide an important help to physicians [5].

- Automation. OARs and other clinical structures in the human body are manually segmented by physicians from medical images, which is difficult, tedious and time consuming [4]. Automating the segmentation process could help tremendously even if it will be only as a pre-step in the diagnosis (used for initial selection of cases or pathologies).

- Finding ROIs. Automatically finding regions could help while preparing for medical procedures or in applying specific procedures on highlighted regions.

- Computer Aided Diagnosis (CADx). To achieve this, a correct delineation of body structures is needed in the pipeline of any CADx systems. Accurate automatic segmentation could be used in non-invasive diagnosis scenarios and could be even deployed online.

- Mass detection. Detecting the mass of organs has as prerequisites a correct segmentation of the organ and the neighbouring surfaces

- Assistance in endoscopic procedures. Automatic segmentation provides help for physicians when executing endoscopic procedures and could be used also in the training phase of the human experts [6].

\section{B. Summary of other Reviews in the same Knowledge Field}

The deep learning knowledge base was described in papers written by Schmidhuber [2], LeCun et al. [3], Benuwa et al. [7] and Voulodimos et al. [8]. More recently, great articles were written by Serre et al. [9] and Alom et al. [10]. 
For a description of deep learning architectures specifically applied in the medical field, we would like to highlight works written by Litjens et al. [11], Shen et al. [1], Hesamian et al. [12], Zhou et al. [13], Ker et al. [14], Taghanaki et al. [15] and $\mathrm{Lu}$ et al. [16]. For details regarding GAN in medical image processing we have an article by Yi et al. [17] and for a review of unsupervised deep learning techniques we have a paper written by Raza et al. [18]. More recently, a comprehensive overview targeted towards multi-organ architectures was written by Lei et al. [4].

\section{The Aim of this Study}

This article discusses the most interesting deep learning architectures and techniques applicable to medical multi-organ segmentation. Targeted to DL-based medical image multiorgan segmentation, there are several objectives that we aimed to fulfil with this article:

- Categorize and summarize the latest research

- Present the most important contributions and identify the current challenges

- Provide an overview of existing medical benchmarks

- Indicate future trends and solutions for the identified challenges

\section{Contents of the Survey}

The paper summarizes over 50 contributions, most of which are more recent than 3 years.

In our process of data searching and gathering, we used several different sources which include arXiv, Google Scholar, PubMed, ISBI, MICCAI or SPIE Medical Imaging. Search keywords included medical segmentation, multi-organ, fully convolutional neural network, and other architectures related to deep learning. The final end-result contains at least 30 articles that describe architectures for single organ segmentation and over 60 articles that detail deep learning techniques for multiorgan delineation.

To make this survey as recent as possible, we have selected works that were mostly published after 2017, while still including older papers that had a big impact in the research field. The most recent date of publication was set to June 1st, 2020, which excluded papers newer than that date.

The bulk of the reviewed works are in Sections II, III and IV and are grouped into three categories - CNNs, FCNs, and hybrid - according to the architecture and which network design is most prominent. The hybrid category has also 3 subsections: GANs, RNNs and fully hybrid approaches. For each architecture classification we presented a small description of the methods and highlighted the most relevant works that were related to multi-organ segmentation. For each included paper we listed the reference, the human structures that were used in training and a summary of their important features and achievements. In Section V we present the most used multiorgan datasets correlated to the human structures that they target. We finalize with a conclusion regarding the future of the research in this subject.

\section{ARChitectures ApPlicABle to MedicAl Multi- ORGAN SEGMENTATION BASED ON CNNS}

A CNN is a sub-genre of deep neural networks [12] that are based on fully connected layers. A layer is made up by more neurons, and each one of these is linked to every neuron from the subsequent layer. A CNN architecture applies a convolution in at least one of its layers. Except for the initial layer, which is linked to the medical image, the input of each layer represents the output of the subsequent layer. Each one of these can perform specific tasks like convolution, pooling, loss calculation while different architectures make use of these layers in differing techniques.

Considering the input image's proportions and the dimension of the convolutional kernels, CNNs can be grouped into three categories. In 2D architectures the medical image is sliced into several 2D images which are fed to the CNN. 2.5D architectures still use 2D kernels, but the network is fed with several patches that are cut from a 3D medical image along the three orthogonal axes. The final category boasts 3D kernels which can extract the full information from a 3D medical image. The major downside of $3 \mathrm{D}$ architectures is the computational and memory requirements which are considered large even using the most up-to-date hardware.

In Table I we present a list of papers that employ CNNs for segmentation in a multi-organ setup. Even though they do not result in a segmentation, papers that present object detection methods in multi-organ scenarios were included in this list. The reason is that they could be used as a pre-step to the actual segmentation by generating regions of interest used to improve the accuracy of the end-result. 
TABLE I. CNN MULTI-ORGAN SEGMENTATION PAPERS

\begin{tabular}{|l|l|l|}
\hline Ref. & Site & Important features \\
\hline$[19]$ & $\begin{array}{l}\text { Brain, Breast and } \\
\text { Cardiac }\end{array}$ & $\begin{array}{l}\text { The authors demonstrate that a 2.5D CNN can be trained in a multi-modality (MRI and CT) scenario to segment tissues } \\
\text { three different human structures. The results were comparable as in using three different architectures for each } \\
\text { segmentation task. }\end{array}$ \\
\hline$[20]$ & Abdomen & $\begin{array}{l}\text { The authors proposed an architecture that segments several abdominal organs using a two-step approach. } \\
\text { Organ localization obtained via a multi-atlas technique followed by training a 3D CNN that classifies the voxels to the } \\
\text { corresponding organ [20]. They also use thresholding as a pre-processing step. }\end{array}$ \\
\hline$[21]$ & $\begin{array}{l}\text { Chest, cardiac, } \\
\text { abdomen }\end{array}$ & $\begin{array}{l}\text { The authors trained a 2.5D CNN that identifies if target human structures are present in input images (CT) [21]. } \\
\text { Bounding boxes can also be placed around the found targeted structures. }\end{array}$ \\
\hline$[22]$ & Brain, abdomen & $\begin{array}{l}\text { The authors propose several methods that can improve the segmentation accuracy: supervised or unsupervised image } \\
\text { enhancement and a novel loss function [22]. }\end{array}$ \\
\hline$[23]$ & Thorax-abdomen & This work presents a 2.5 CNN trained for localization of several human structures in CT images [23]. \\
\hline$[24]$ & Pelvic organs & $\begin{array}{l}\text { The authors propose a novel hierarchical dilated CNN. The novelty is that they propose a multi-scale architecture } \\
\text { comprised of several modules working with different resolutions [24]. }\end{array}$ \\
\hline$[25]$ & Torso - 17 organs & The authors propose an architecture for organ localization and 3D bounding boxes generation [25]. \\
\hline$[26]$ & Head and neck & The article proposes a multi-organ segmentation architecture that cascades three CNNs followed by majority voting [26]. \\
\hline$[27]$ & Head and body & $\begin{array}{l}\text { The authors propose an architecture for organ localization based on a 3D CNN that also improves the localization } \\
\text { performance on small organs [27]. }\end{array}$ \\
\hline
\end{tabular}

III. ARChiteCtures ApPlicable to MedicAl MultiORGAN SEGMENTATION BASED ON FCNS

CNNs can classify each individual voxel from a medical image, but this approach has a huge drawback. Because the neighbouring patches on which convolutions are calculated have overlapping voxels, the same calculations are done multiple times with performance penalties. To counter this major issue, Long et al. [28] proposed the "Fully convolutional network" where the size of the predicted image is increased to match the size of the input image by using a transposed convolution layer. Ronneberger et al. [29] proposed the U-Net network that has a contracting path with layers that include convolutions, max pooling and Rectified Linear Unit (RELU) [30] and an expanding path that involves up-convolutions and concatenations with high-resolution features from the contracting path [29]. Çiçek et al. [31] implemented the first 3D U-Net design while Milletari et al. [32] improved the U-Net architecture by adding residual blocks and a dice loss layer.

In Table II we present a list of papers that employ FCNs for segmentation in a multi-organ setup.

TABLE II. FCN MULTI-ORGAN SEGMENTATION PAPERS

\begin{tabular}{|c|c|c|}
\hline Ref. & Site & Important features \\
\hline [33] & Liver and heart & $\begin{array}{l}\text { The authors propose a 3D FCN enhanced by a deep supervision technique [33]. The architecture is validated against heart } \\
\text { and liver datasets (not a full-blown multi-organ implementation). }\end{array}$ \\
\hline [6] & Abdomen & $\begin{array}{l}\text { The article proposed an approach on segmenting } 4 \text { abdominal organs using an FCN that employs "dilated convolution units } \\
\text { with dense skip connections" [6]. }\end{array}$ \\
\hline$[34]$ & Abdomen & $\begin{array}{l}\text { In this article the authors prove that a "multi-class 3D FCN trained for seven abdominal structures can achieve competitive } \\
\text { segmentation results, while avoiding the need training organ-specific models" [34]. } \\
\text { They proposed an architecture comprised of two FCNs, with the first delineating a candidate region, while the later uses } \\
\text { that as input for the final segmentation. }\end{array}$ \\
\hline$[35]$ & $\begin{array}{l}\text { Esophagus, Trachea, } \\
\text { Heart, Aorta }\end{array}$ & $\begin{array}{l}\text { The authors propose "two collaborative architectures to jointly segment multiple organs" [35]. The first network will learn } \\
\text { anatomical constraints employing also conditional random fields, while its output will be used by the second network for } \\
\text { guiding and refining the segmentation. }\end{array}$ \\
\hline$[36]$ & Liver, spleen, kidneys & $\begin{array}{l}\text { The authors propose a deep 3D FCN for organ segmentation that is enhanced using a "time-implicit multi-phase evolution } \\
\text { method" [36]. }\end{array}$ \\
\hline [37] & $\begin{array}{l}\text { Torso and special } \\
\text { regions: lumen and } \\
\text { stomach content }\end{array}$ & $\begin{array}{l}\text { The authors propose a } 2.5 \mathrm{D} \text { FCN architecture trained on CTs. The algorithm uses a fusion method for the final 3D } \\
\text { segmentation. They summarize the algorithm as "multiple 2D proposals followed by } 3 \mathrm{D} \text { integration" [37]. }\end{array}$ \\
\hline$[38]$ & Liver, Left kidney & $\begin{array}{l}\text { In this paper, the authors propose an improvement of their previous segmentation architectures by adding an organ } \\
\text { localization module [38]. }\end{array}$ \\
\hline [39] & Gastro-intestinal tract & $\begin{array}{l}\text { The authors present an implementation of a Dense V-Net architecture in a multi-organ setup while showing that their } \\
\text { proposed "dense connections and the multi-scale structure" [39] produce better segmentation results. }\end{array}$ \\
\hline [40] & Abdomen & $\begin{array}{l}\text { The paper describes an implementation of a 3D U-Net for multi-organ CT segmentation [40]. The authors obtained a } \\
\text { combined dice of } 89.3 \% \text { in testing } 7 \text { organs. }\end{array}$ \\
\hline
\end{tabular}




\begin{tabular}{|c|c|c|}
\hline Ref. & Site & Important features \\
\hline [41] & Abdomen & $\begin{array}{l}\text { The authors present an architecture that is based on a "multi-scale pyramid of stacked 3D FCNs" [41]. The results are } \\
\text { obtained by taking the predictions of a lower-resolution 3D FCN up-sampling, cropping them and afterwards concatenating } \\
\text { them with the inputs of a 3D FCN that utilizes a higher resolution which will generate a final segmentation. }\end{array}$ \\
\hline$[42]$ & Abdomen & $\begin{array}{l}\text { The authors argue that the results of multi-organ segmentation using FCNs depend on the architecture, but also are heavily } \\
\text { influenced by the chosen loss function [42]. They also evaluate the loss function's influence in multi-organ segmentation } \\
\text { scenarios. }\end{array}$ \\
\hline [43] & Abdomen & $\begin{array}{l}\text { The authors propose a cascaded approach that uses two 3D FCNs. The first architecture defines a candidate region, while } \\
\text { the second focuses on the details and provides the final segmentation. The authors argue that their "approach reduces the } \\
\text { number of voxels the second FCN must classify to } \sim 10 \% \text { " [43]. }\end{array}$ \\
\hline [44] & Torso & $\begin{array}{l}\text { The paper presents three 3D FCN architectures and surveys their results of multi-organ segmentation in the human torso. } \\
\text { The dice scores average between } 0.91 \text { and } 0.98 \text { for } 6 \text { covered organs. }\end{array}$ \\
\hline [45] & Head and neck & $\begin{array}{l}\text { The authors present an architecture based on a 3D U-Net that is tested against a head and neck dataset. The results were } \\
\text { mixed, with fair segmentation scores for } 7 \text { organs out of } 11 \text {, but with low results for the other organs. }\end{array}$ \\
\hline [46] & Brain, Abdomen & $\begin{array}{l}\text { The authors propose an FCN architecture [46] that outperforms the initial U-Net implementation in several segmentation } \\
\text { tasks for brain or abdomen. The results have a dice percentage between } 83.42 \% \text { and } 96.57 \% \text { for several abdomen organs. }\end{array}$ \\
\hline [47] & Chest & The authors propose an architecture based on two cascaded networks. \\
\hline [48] & Abdomen & $\begin{array}{l}\text { The authors present a novel architecture that improves the segmentation using a transfer learning scheme. 3D U-Nets are } \\
\text { used in a general approach or single organ approach with transfer learning between them. Furthermore, probabilistic atlases } \\
\text { are used to estimate the location of the organs. }\end{array}$ \\
\hline [49] & Abdomen & $\begin{array}{l}\text { The authors present an architecture for segmentation in a multi-organ scenario consisting of a " } 2 \mathrm{D} \text { U-Net localization } \\
\text { network and a 3D U-Net segmentation network" [49]. Compared to other architectures, the authors results are better for } \\
\text { several organs like prostate and bladder. }\end{array}$ \\
\hline$[50]$ & Abdomen & $\begin{array}{l}\text { The authors propose a two-step architecture. The first step contains 2D networks with reverse connections that detect } \\
\text { features. These features are afterwards merged with the original image to "enhance the discriminative information for the } \\
\text { target organs" [50] and are used as input for the final segmentation network. }\end{array}$ \\
\hline$[51]$ & Gland & The paper describes two Dense U-Nets used for segmentation of several gland types. \\
\hline$[52]$ & Abdomen & $\begin{array}{l}\text { The authors propose a multi-organ segmentation architecture based on 3D convolution [52]. Their design obtained an } \\
\text { average Dice score of } 83.7 \% \text { for } 6 \text { abdominal organs in their targeted dataset. }\end{array}$ \\
\hline [53] & Thorax & $\begin{array}{l}\text { The authors propose an architecture where a 3D U-Net localizes each target organ. Afterwards, cropped images with one } \\
\text { organ serve as input to several individual 3D U-Net segmentation networks and as a final step the individual results are } \\
\text { merged for a global segmentation result. }\end{array}$ \\
\hline$[54]$ & Thorax and abdomen & $\begin{array}{l}\text { The paper describes in detail the SegTHOR [54] multi-organ dataset and present a segmentation framework based on U- } \\
\text { Net. }\end{array}$ \\
\hline$[55]$ & Thorax and abdomen & $\begin{array}{l}\text { The paper proposes an architecture for segmentation of the SegTHOR [54] multi-organ dataset that consists of two 3D V- } \\
\text { Net working on different resolutions (one for organ localization and one for segmentation refinement). Their approach } \\
\text { ranked first in the initial phase of the SEGTHOR challenge. }\end{array}$ \\
\hline$[56]$ & Thorax and abdomen & $\begin{array}{l}\text { The authors propose an improvement to the U-Net and obtain a "uniform U-like encoder-decoder segmentation } \\
\text { architecture" [56]. The architecture ranked second on the initial phase of the SEGTHOIR challenge. }\end{array}$ \\
\hline [57] & Thorax and abdomen & $\begin{array}{l}\text { The authors propose a simplified version of the Dense V-net model with postprocessing that improve the organ } \\
\text { segmentation results. }\end{array}$ \\
\hline$[58]$ & Thorax & $\begin{array}{l}\text { The paper proposes a multi-organ segmentation architecture that contains "dilated convolutions and aggregated residual } \\
\text { connections in a U-Net styled network" [58]. }\end{array}$ \\
\hline [59] & Abdomen, torso & The paper proposes a 3D U-Net like architecture [59] that is validated on 5 different organs. \\
\hline$[60]$ & Abdomen & $\begin{array}{l}\text { The authors propose a multi-class segmentation architecture based on U-Net [60]. Their design has similar results to other } \\
\text { approaches on } 4 \text { organs but with superior dice scores for the intestine. }\end{array}$ \\
\hline$[61]$ & Abdomen & The authors propose a 3D U-Net architecture tested in a multi-organ segmentation scenario. \\
\hline$[62]$ & Abdomen & $\begin{array}{l}\text { The authors propose an architecture consisting of a 3D U-Net that is enhanced by graph-cut post-processing [62] tested in a } \\
\text { multi-organ segmentation scenario. }\end{array}$ \\
\hline$[63]$ & Abdomen & $\begin{array}{l}\text { The authors present a "pyramid-input pyramid-output" [63] architecture that can be trained in a multi-scale and partially } \\
\text { labeled scenario. In order to discriminate the features in differing scales, they designed an "adaptive weighting layer to fuse } \\
\text { the outputs in an automatic fashion" [63] }\end{array}$ \\
\hline
\end{tabular}




\section{ARChitectures ApPlicable to Medical Multi- ORGAN SEGMENTATION BASED ON HYBRID METHODS}

As the DL field is expanding, new and exciting network architectures are developed. At the same time, the possibilities of improving the existing segmentation networks are shrinking. Therefore, to overcome these challenges, hybrid approaches are used more extensively. These hybrid methods involve using several network designs in the same architecture serving different functional purposes. We have divided the hybrid approaches into segmentation architectures enriched with GANs, enriched with RNNs and fully hybrid approaches.

\section{A. Hybrid Methods Employing GANs}

A GAN is a type of machine learning network designed by Goodfellow et al. [64]. These networks are taught to be able to generate new data that shares the same characteristics as a provided initial training set. In Table III we present a list of papers that propose GAN based hybrid multi-organ architectures.

\section{B. Hybrid Methods Employing RNNs}

A Recurrent Neural Network (RNN) is a type of machine learning network that generalizes the feedforward neural network architecture and has hidden states that act as an internal memory. Empowered with these connections the RNNs can memorize the patterns from previous inputs. These architectures are applied mostly to time series predictions or speech recognition. But because medical images are usually comprised of multiple adjacent slices with correlating information between them, RNNs can be employed in hybrid scenarios to improve the segmentation results. In Table IV we present a list of papers that propose RNN based hybrid multiorgan architectures.

TABLE III. GAN MULTI-ORGAN SEGMENTATION PAPERS

\begin{tabular}{|l|l|l|}
\hline Ref. & Site & Important features \\
\hline [65] & $\begin{array}{l}\text { Brain, } \\
\text { liver, } \\
\text { cells }\end{array}$ & $\begin{array}{l}\text { The authors propose an architecture made by "a } \\
\text { generative, a discriminative, and a refinement network" } \\
\text { [65] based on U-Net. The final semantic segmentation } \\
\text { masks are composed by the output of the three } \\
\text { networks. }\end{array}$ \\
\hline [66] & Thorax & $\begin{array}{l}\text { The paper describes an architecture that trains a set of } \\
\text { generator networks (based on U-Net) and a set of } \\
\text { discriminators (based on FCNs) "The generator and } \\
\text { discriminator compete against each other in an } \\
\text { adversarial learning process to produce the optimal } \\
\text { segmentation map of multiple organs" [66]. }\end{array}$ \\
\hline [67] & Thorax & $\begin{array}{l}\text { The authors propose a hybrid architecture that first } \\
\text { generates a "global localization map by minimizing a } \\
\text { reconstruction error within an adversarial framework } \\
\text { [67]. Afterwards, the localization map guides an FNC } \\
\text { for multi-organ segmentation. }\end{array}$ \\
\hline [68] & Abdomen & $\begin{array}{l}\text { The authors present a hybrid architecture that combines } \\
\text { GAN based image synthesis methods with a deep } \\
\text { attention strategy that learns discriminative features for } \\
\text { organ segmentation. }\end{array}$ \\
\hline [69] & Abdomen & $\begin{array}{l}\text { The paper describes a hybrid architecture that combines } \\
\text { cascaded convolutional networks with adversarial } \\
\text { networks to alleviate data scarcity limitations. }\end{array}$ \\
\hline
\end{tabular}

TABLE IV. RNN MULTI-ORGAN SEGMENTATION PAPERS

\begin{tabular}{|c|c|c|}
\hline Ref. & Site & Important features \\
\hline [70] & $\begin{array}{l}\text { Optic disc, } \\
\text { cell nuclei, } \\
\text { left atrium }\end{array}$ & $\begin{array}{l}\text { The authors present a hybrid architecture that } \\
\text { combines a CNN with an RNN. }\end{array}$ \\
\hline [71] & $\begin{array}{l}\text { Abdomen - } \\
\text { small organs }\end{array}$ & $\begin{array}{l}\text { This paper presents an architecture in which a } \\
\text { recurrent module "repeatedly converts the } \\
\text { segmentation probability map from the previous } \\
\text { iteration as spatial weights and applies these } \\
\text { weights to the current iteration" [71]. }\end{array}$ \\
\hline [72] & $\begin{array}{l}\text { Blood vessel, } \\
\text { skin cancer, } \\
\text { lungs }\end{array}$ & $\begin{array}{l}\text { The article presents a hybrid architecture based on } \\
\text { U-Net and RNN where the "feature accumulation } \\
\text { with recurrent residual convolutional layers" [72] } \\
\text { provides better segmentation end results. }\end{array}$ \\
\hline [73] & Abdomen & $\begin{array}{l}\text { The paper proposes an attention gate model that } \\
\text { can be integrated into neural networks." Models } \\
\text { trained with AGs implicitly learn to suppress } \\
\text { irrelevant regions in an input image" [73]. }\end{array}$ \\
\hline [74] & $\begin{array}{l}\text { Vertebrae, } \\
\text { liver }\end{array}$ & $\begin{array}{l}\text { The authors present a hybrid architecture that } \\
\text { consists of a U-Net-like network enhanced with } \\
\text { bidirectional C-LSTM [74]. }\end{array}$ \\
\hline
\end{tabular}

\section{Fully Hybrid and Generic Segmentation Improvement Methods}

In Table $\mathrm{V}$ we present hybrid methods that do not fit in any previous category and generic segmentation improvement methods in multi-organ scenarios.

TABLE V. Hybrid Multi-ORgan SEgmentation PAPERS

\begin{tabular}{|c|c|c|}
\hline Ref. & Site & Important features \\
\hline [75] & $\begin{array}{l}\text { Torso and } \\
\text { abdomen }\end{array}$ & $\begin{array}{l}\text { The authors present a sample selection method } \\
\text { [75] that improves the training of neural networks. } \\
\text { The method is tested in a multi-organ } \\
\text { segmentation scenario. }\end{array}$ \\
\hline [76] & Abdomen & $\begin{array}{l}\text { The authors investigate the "effectiveness of } \\
\text { learning from multiple modalities to improve the } \\
\text { segmentation accuracy" [76]. }\end{array}$ \\
\hline [77] & Abdomen & $\begin{array}{l}\text { The authors propose an architecture in which an } \\
\text { initial model is trained on annotated data to } \\
\text { generate pseudo labels that enrich the training data } \\
\text { for a second model that will do a final } \\
\text { segmentation. }\end{array}$ \\
\hline [78] & Abdomen & $\begin{array}{l}\text { The authors propose an architecture that } \\
\text { incorporates anatomical domain knowledge on } \\
\text { abdominal organ sizes to guide and improve the } \\
\text { training process. }\end{array}$ \\
\hline [79] & Retina, lungs & $\begin{array}{l}\text { The paper describes an architecture that "embeds } \\
\text { edge-attention representations to guide the process } \\
\text { of segmentation" [79]. }\end{array}$ \\
\hline [80] & $\begin{array}{l}\text { Heart, gland, } \\
\text { lymph node }\end{array}$ & $\begin{array}{l}\text { The authors propose an architecture that firstly } \\
\text { decomposes the segmentation problem into several } \\
\text { sub-problems, then applies DL modules onto each } \\
\text { sub-problem and lastly integrates the results to } \\
\text { obtain the final segmentation. }\end{array}$ \\
\hline [81] & $\begin{array}{l}\text { Abdomen, } \\
\text { heart, brain }\end{array}$ & $\begin{array}{l}\text { The authors present an architecture that tries "to } \\
\text { integrate local features with their corresponding } \\
\text { global dependencies" [81] by using a guided self- } \\
\text { attention mechanism. }\end{array}$ \\
\hline
\end{tabular}




\section{ArChitectures Applicable to Medical Multi- ORGAN SEGMENTATION BASED ON HYBRID METHODS}

There are multiple collaborative initiatives with medical organizations to obtain better and larger datasets usable for organ segmentation. But despite all these efforts, the amount of annotated data that is at the disposal of DL scientists is still low. There are solutions in combining several datasets of parts of the human body, but different modalities or scales reduce considerably their usage in multi-organ segmentation scenarios. In Table VI we present several datasets that try to overcome these challenges and are usable in multi-organ validation of segmentation architectures.

TABLE VI. MULTI-ORGAN DATASETS

\begin{tabular}{|c|c|c|c|}
\hline Year & Dataset & Modality & Organ \\
\hline 2015 & $\begin{array}{l}\text { "MICCAI Multi-Atlas Labeling } \\
\text { Beyond the Cranial Vault" [82] }\end{array}$ & $\mathrm{CT}$ & Abdomen \\
\hline 2015 & $\begin{array}{l}\text { "MICCAI Challenge - Head and } \\
\text { Neck Auto Segmentation } \\
\text { Challenge" [83] }\end{array}$ & CT & $\begin{array}{l}\text { Head and } \\
\text { Neck }\end{array}$ \\
\hline 2017 & $\begin{array}{l}\text { "AAPM Thoracic Auto- } \\
\text { segmentation Challenge" [84] }\end{array}$ & $\mathrm{CT}$ & Thorax \\
\hline 2018 & $\begin{array}{l}\text { "Medical Segmentation Decathlon" } \\
{[85]}\end{array}$ & CT \& MRI & $\begin{array}{l}\text { Head, } \\
\text { Thorax and } \\
\text { Abdomen }\end{array}$ \\
\hline 2019 & $\begin{array}{l}\text { "CHAOS - Combined (CT-MR) } \\
\text { Healthy Abdominal Organ } \\
\text { Segmentation" [86] }\end{array}$ & CT \& MRI & Abdomen \\
\hline 2019 & $\begin{array}{l}\text { "SegTHOR Challenge: } \\
\text { Segmentation of Thoracic Organs } \\
\text { at Risk in CT Images" [54] }\end{array}$ & $\mathrm{CT}$ & Thorax \\
\hline 2019 & $\begin{array}{l}\text { "Annotations for Body Organ } \\
\text { Localization based on MICCAI } \\
\text { LITS Dataset" [87] }\end{array}$ & $\mathrm{CT}$ & Abdomen \\
\hline
\end{tabular}

\section{Final CONCLUSIONS}

This paper is an overview of deep learning methods in medical multi-organ segmentation. Based on most of the surveyed works, FCNs are the most used architectures used to perform multi-organ automatic delineating. As the amount of research related to FCNs is huge, the possibilities to improve them is dwindling. So, more recently, hybrid methods, be it with the use of GANs, RNNs or completely new architectures are gaining much more attention. We speculate that in the future the number of available datasets will grow, so the usage of FCNs or hybrid networks will become more straightforward. Another un-charted territory is the usage of more intelligent semi-supervised methods, the usage of fully unsupervised methods or reinforcement learning.

\section{REFERENCES}

[1] D. Shen, G. Wu, and H.-I. Suk, "Deep learning in medical image analysis," Annu. Rev. Biomed. Eng., vol. 19, no. 1, pp. 221-248, Jun. 2017, doi: 10.1146/annurev-bioeng-071516-044442.

[2] J. Schmidhuber, "Deep learning in neural networks: An overview," Neural Networks, vol. 61, pp. 85-117, Jan. 2015, doi: 10.1016/j.neunet.2014.09.003.

[3] Y. LeCun, Y. Bengio, and G. Hinton, "Deep learning," Nature, vol. 521, no. 7553, pp. 436-444, May 2015, doi: 10.1038/nature14539.
[4] Y. Lei et al., "Deep learning in multi-organ segmentation," arXiv:2001.10619 [physics], Jan. 2020. Available: http://arxiv.org/abs/2001.10619.

[5] B. Ibragimov and L. Xing, "Segmentation of organs-at-risks in head and neck CT images using convolutional neural networks," Med. Phys., vol. 44, no. 2, pp. 547-557, Feb. 2017, doi: 10.1002/mp. 12045.

[6] E. Gibson et al., "Towards image-guided pancreas and biliary endoscopy: automatic multi-organ segmentation on abdominal CT with dense dilated networks," in Medical Image Computing and Computer Assisted Intervention - MICCAI 2017, vol. 10433, M. Descoteaux, L. Maier-Hein, A. Franz, P. Jannin, D. L. Collins, and S. Duchesne, Eds. Cham: Springer International Publishing, 2017, pp. 728-736.

[7] B. B. Benuwa, Y. Z. Zhan, B. Ghansah, D. K. Wornyo, and F. Banaseka Kataka, "A review of deep machine learning," JERA, vol. 24, pp. 124136, Jun. 2016, doi: 10.4028/www.scientific.net/JERA.24.124.

[8] A. Voulodimos, N. Doulamis, A. Doulamis, and E. Protopapadakis, "Deep learning for computer vision: A brief review," Computational Intelligence and Neuroscience, vol. 2018, pp. 1-13, 2018, doi: $10.1155 / 2018 / 7068349$.

[9] T. Serre, "Deep learning: The good, the bad, and the ugly," Annu. Rev. Vis. Sci., vol. 5, no. 1, pp. 399-426, Sep. 2019, doi: 10.1146/annurevvision-091718-014951.

[10] M. Z. Alom et al., "A state-of-the-art survey on deep learning theory and architectures," Electronics, vol. 8, no. 3, p. 292, Mar. 2019, doi: 10.3390/electronics8030292.

[11] G. Litjens et al., "A survey on deep learning in medical image analysis," Medical Image Analysis, vol. 42, pp. 60-88, Dec. 2017, doi: 10.1016/j.media.2017.07.005.

[12] M. H. Hesamian, W. Jia, X. He, and P. Kennedy, "Deep learning techniques for medical image segmentation: Achievements and challenges,” J Digit Imaging, vol. 32, no. 4, pp. 582-596, Aug. 2019, doi: 10.1007/s10278-019-00227-x.

[13] T. Zhou, S. Ruan, and S. Canu, "A review: Deep learning for medical image segmentation using multi-modality fusion," Array, vol. 3-4, p. 100004, Sep. 2019, doi: 10.1016/j.array.2019.100004.

[14] J. Ker, L. Wang, J. Rao, and T. Lim, "Deep learning applications in medical image analysis," IEEE Access, vol. 6, pp. 9375-9389, 2018, doi: 10.1109/ACCESS.2017.2788044.

[15] S. A. Taghanaki, K. Abhishek, J. P. Cohen, J. Cohen-Adad, and G. Hamarneh, "Deep semantic segmentation of natural and medical images: A review," arXiv:1910.07655 [cs, eess], Jun. 2020. Available: http://arxiv.org/abs/1910.07655.

[16] L. Lu, X. Wang, G. Carneiro, and L. Yang, "Deep learning and convolutional neural networks for medical imaging and clinical informatics,". 2019.

[17] X. Yi, E. Walia, and P. Babyn, "Generative adversarial network in medical imaging: A review," Medical Image Analysis, vol. 58, p. 101552, Dec. 2019, doi: 10.1016/j.media.2019.101552.

[18] K. Raza and N. K. Singh, "A tour of unsupervised deep learning for medical image analysis," arXiv:1812.07715 [cs, eess], Dec. 2018. Available: http://arxiv.org/abs/1812.07715.

[19] P. Moeskops et al., "Deep learning for multi-task medical image segmentation in multiple modalities," arXiv:1704.03379 [cs], vol. 9901, pp. 478-486, 2016, doi: 10.1007/978-3-319-46723-8_55.

[20] M. Larsson, Y. Zhang, and F. Kahl, "Robust abdominal organ segmentation using regional convolutional neural networks," in Image Analysis, vol. 10270, P. Sharma and F. M. Bianchi, Eds. Cham: Springer International Publishing, 2017, pp. 41-52.

[21] B. D. de Vos, J. M. Wolterink, P. A. de Jong, T. Leiner, M. A. Viergever, and I. Isgum, "ConvNet-based localization of anatomical structures in 3-D medical images," IEEE Trans. Med. Imaging, vol. 36, no. 7, pp. 1470-1481, Jul. 2017, doi: 10.1109/TMI.2017.2673121.

[22] G. Wang et al., "Interactive medical image segmentation using deep learning with image-specific fine-tuning," IEEE Trans. Med. Imaging, vol. 37, no. 7, pp. 1562-1573, Jul. 2018, doi: 10.1109/TMI.2018.2791721.

[23] G. E. Humpire-Mamani, A. A. A. Setio, B. van Ginneken, and C. Jacobs, "Efficient organ localization using multi-label convolutional 
neural networks in thorax-abdomen CT scans," Phys. Med. Biol., vol. 63, no. 8, p. 085003, Apr. 2018, doi: 10.1088/1361-6560/aab4b3.

[24] S. Zhou et al., "Fine-grained segmentation using hierarchical dilated neural networks," in Medical Image Computing and Computer Assisted Intervention - MICCAI 2018, vol. 11073, A. F. Frangi, J. A. Schnabel, C. Davatzikos, C. Alberola-López, and G. Fichtinger, Eds. Cham: Springer International Publishing, 2018, pp. 488-496.

[25] X. Zhou et al., "Automatic anatomy partitioning of the torso region on CT images by using a deep convolutional network with majority voting," in Medical Imaging 2019: Computer-Aided Diagnosis, San Diego, United States, Mar. 2019, p. 34, doi: 10.1117/12.2512651.

[26] T. Zhong, X. Huang, F. Tang, S. Liang, X. Deng, and Y. Zhang, "Boosting-based cascaded convolutional neural networks for the segmentation of CT organs-at-risk in nasopharyngeal carcinoma," Med. Phys., vol. 46, no. 12, pp. 5602-5611, Dec. 2019, doi: $10.1002 / \mathrm{mp} .13825$.

[27] X. Xu, F. Zhou, B. Liu, D. Fu, and X. Bai, "efficient multiple organ localization in ct image using $3 \mathrm{~d}$ region proposal network," IEEE Trans. Med. Imaging, vol. 38, no. 8, pp. 1885-1898, Aug. 2019, doi: 10.1109/TMI.2019.2894854.

[28] J. Long, E. Shelhamer, and T. Darrell, "Fully convolutional networks for semantic segmentation," in 2015 IEEE Conference on Computer Vision and Pattern Recognition (CVPR), Boston, MA, USA, Jun. 2015, pp. 3431-3440, doi: 10.1109/CVPR.2015.7298965.

[29] O. Ronneberger, P. Fischer, and T. Brox, "U-Net: Convolutional networks for biomedical image segmentation," arXiv:1505.04597 [cs], May 2015. Available: http://arxiv.org/abs/1505.04597.

[30] V. Nair and G. E. Hinton, "Rectified linear units improve restricted boltzmann machines," in Proceedings of the 27th International Conference on International Conference on Machine Learning, Madison, WI, USA, 2010, pp. 807-814.

[31] Ö. Çiçek, A. Abdulkadir, S. S. Lienkamp, T. Brox, and O. Ronneberger, "3D U-Net: Learning dense volumetric segmentation from sparse annotation," arXiv:1606.06650 [cs], Jun. 2016. Available: http://arxiv.org/abs/1606.06650.

[32] F. Milletari, N. Navab, and S.-A. Ahmadi, "V-Net: Fully convolutional neural networks for volumetric medical image segmentation," arXiv:1606.04797 [cs], Jun. 2016. Available: http://arxiv.org/abs/1606.04797.

[33] Q. Dou et al., "3D deeply supervised network for automated segmentation of volumetric medical images," Medical Image Analysis, vol. 41, pp. 40-54, Oct. 2017, doi: 10.1016/j.media.2017.05.001.

[34] H. R. Roth et al., "Hierarchical 3D fully convolutional networks for multi-organ segmentation," arXiv:1704.06382 [cs], Apr. 2017. Available: http://arxiv.org/abs/1704.06382.

[35] R. Trullo, C. Petitjean, D. Nie, D. Shen, and S. Ruan, "Joint segmentation of multiple thoracic organs in ct images with two collaborative deep architectures," in Deep Learning in Medical Image Analysis and Multimodal Learning for Clinical Decision Support, vol. 10553, M. J. Cardoso, T. Arbel, G. Carneiro, T. Syeda-Mahmood, J. M. R. S. Tavares, M. Moradi, A. Bradley, H. Greenspan, J. P. Papa, A. Madabhushi, J. C. Nascimento, J. S. Cardoso, V. Belagiannis, and Z. Lu, Eds. Cham: Springer International Publishing, 2017, pp. 21-29.

[36] P. Hu, F. Wu, J. Peng, Y. Bao, F. Chen, and D. Kong, "Automatic abdominal multi-organ segmentation using deep convolutional neural network and time-implicit level sets," Int J CARS, vol. 12, no. 3, pp. 399-411, Mar. 2017, doi: 10.1007/s11548-016-1501-5.

[37] X. Zhou, R. Takayama, S. Wang, T. Hara, and H. Fujita, "Deep learning of the sectional appearances of 3D CT images for anatomical structure segmentation based on an FCN voting method," Med. Phys., vol. 44, no. 10, pp. 5221-5233, Oct. 2017, doi: 10.1002/mp.12480.

[38] X. Zhou, R. Takayama, S. Wang, X. Zhou, T. Hara, and H. Fujita, "Automated segmentation of $3 \mathrm{D}$ anatomical structures on CT images by using a deep convolutional network based on end-to-end learning approach," Orlando, Florida, United States, Feb. 2017, p. 1013324, doi: $10.1117 / 12.2254201$

[39] E. Gibson et al., "Automatic multi-organ segmentation on abdominal CT with dense v-networks," IEEE Trans. Med. Imaging, vol. 37, no. 8, pp. 1822-1834, Aug. 2018, doi: 10.1109/TMI.2018.2806309.
[40] H. Roth et al., "Deep learning and its application to medical image segmentation," ArXiv, vol. abs/1803.08691, 2018.

[41] H. R. Roth et al., "A multi-scale pyramid of 3D fully convolutional networks for abdominal multi-organ segmentation," arXiv:1806.02237 [cs], Jun. 2018. Available: http://arxiv.org/abs/1806.02237.

[42] C. Shen et al., "On the influence of Dice loss function in multi-class organ segmentation of abdominal CT using 3D fully convolutional networks," arXiv:1801.05912 [cs], Jan. 2018. Available: http://arxiv.org/abs/1801.05912.

[43] H. R. Roth et al., "An application of cascaded 3D fully convolutional networks for medical image segmentation," Computerized Medical Imaging and Graphics, vol. 66, pp. 90-99, Jun. 2018, doi: 10.1016/j.compmedimag.2018.03.001.

[44] S. Chen et al., "Automatic multi-organ segmentation in dual energy CT using 3D fully convolutional network," 2018.

[45] W. van Rooij, M. Dahele, H. Ribeiro Brandao, A. R. Delaney, B. J. Slotman, and W. F. Verbakel, "Deep learning-based delineation of head and neck organs at risk: geometric and dosimetric evaluation," International Journal of Radiation Oncology*Biology*Physics, vol. 104, no. 3, pp. 677-684, Jul. 2019, doi: 10.1016/j.ijrobp.2019.02.040.

[46] L. Chen, P. Bentley, K. Mori, K. Misawa, M. Fujiwara, and D. Rueckert, "DRINet for medical image segmentation," IEEE Trans. Med. Imaging, vol. 37, no. 11, pp. 2453-2462, Nov. 2018, doi: 10.1109/TMI.2018.2835303.

[47] G. González, G. R. Washko, and R. San José Estépar, "Multi-structure segmentation from partially labeled datasets. Application to body composition measurements on CT scans," in Image Analysis for Moving Organ, Breast, and Thoracic Images, vol. 11040, D. Stoyanov, Z. Taylor, B. Kainz, G. Maicas, R. R. Beichel, A. Martel, L. Maier-Hein, K. Bhatia, T. Vercauteren, O. Oktay, G. Carneiro, A. P. Bradley, J. Nascimento, H. Min, M. S. Brown, C. Jacobs, B. Lassen-Schmidt, K. Mori, J. Petersen, R. San José Estépar, A. Schmidt-Richberg, and C. Veiga, Eds. Cham: Springer International Publishing, 2018, pp. 215224.

[48] H. Kakeya, T. Okada, and Y. Oshiro, "3D U-JAPA-Net: Mixture of convolutional networks for abdominal multi-organ CT segmentation," in Medical Image Computing and Computer Assisted Intervention MICCAI 2018, vol. 11073, A. F. Frangi, J. A. Schnabel, C. Davatzikos, C. Alberola-López, and G. Fichtinger, Eds. Cham: Springer International Publishing, 2018, pp. 426-433.

[49] A. Balagopal et al., "Fully automated organ segmentation in male pelvic CT images," Phys. Med. Biol., vol. 63, no. 24, p. 245015, Dec. 2018, doi: 10.1088/1361-6560/aaf11c.

[50] Y. Wang, Y. Zhou, W. Shen, S. Park, E. K. Fishman, and A. L. Yuille, "Abdominal multi-organ segmentation with organ-attention networks and statistical fusion," Medical Image Analysis, vol. 55, pp. 88-102, Jul. 2019, doi: 10.1016/j.media.2019.04.005.

[51] T. Binder, E. M. Tantaoui, P. Pati, R. Catena, A. Set-Aghayan, and M. Gabrani, "Multi-organ gland segmentation using deep learning," Front. Med., vol. 6, p. 173, Aug. 2019, doi: 10.3389/fmed.2019.00173.

[52] M. P. Heinrich, O. Oktay, and N. Bouteldja, "OBELISK-Net: Fewer layers to solve 3D multi-organ segmentation with sparse deformable convolutions," Medical Image Analysis, vol. 54, pp. 1-9, May 2019, doi: 10.1016/j.media.2019.02.006.

[53] X. Feng, K. Qing, N. J. Tustison, C. H. Meyer, and Q. Chen, "Deep convolutional neural network for segmentation of thoracic organs-at-risk using cropped 3D images," Med. Phys., vol. 46, no. 5, pp. 2169-2180, May 2019, doi: 10.1002/mp.13466.

[54] Z. Lambert, C. Petitjean, B. Dubray, and S. Ruan, "SegTHOR: Segmentation of thoracic organs at risk in CT images," arXiv:1912.05950 [cs, eess], Dec. 2019. Available: http://arxiv.org/abs/1912.05950.

[55] M. Han et al., "Segmentation of CT thoracic organs by multi-resolution VB-nets," 2019.

[56] T. He, J. Hu, Y. Song, J. Guo, and Z. Yi, "Multi-task learning for the segmentation of organs at risk with label dependence," Medical Image Analysis, vol. 61, p. 101666, Apr. 2020, doi: 10.1016/j.media.2020.101666. 
[57] M. Feng, W. Huang, Y. Wang, and Y. Xie, "Multi-organ segmentation using simplified dense V-net with post-processing," 2019.

[58] S. Vesal, N. Ravikumar, and A. Maier, "A 2D dilated residual U-Net for multi-organ segmentation in thoracic CT," arXiv:1905.07710 [cs, eess], May 2019. Available: http://arxiv.org/abs/1905.07710.

[59] C. Huang, H. Han, Q. Yao, S. Zhu, and S. K. Zhou, "3D U\$^2\$-Net: A 3D universal U-Net for multi-domain medical image segmentation," arXiv:1909.06012 [cs, eess], Sep. 2019. Available: http://arxiv.org/abs/1909.06012.

[60] Z. Liu et al., "Segmentation of organs-at-risk in cervical cancer CT images with a convolutional neural network," Physica Medica, vol. 69, pp. 184-191, Jan. 2020, doi: 10.1016/j.ejmp.2019.12.008.

[61] J. Léger, E. Brion, P. Desbordes, C. De Vleeschouwer, J. A. Lee, and B. Macq, "Cross-domain data augmentation for deep-learning-based male pelvic organ segmentation in cone beam CT," Applied Sciences, vol. 10, no. 3, p. 1154, Feb. 2020, doi: 10.3390/app10031154.

[62] H. Kim et al., "Abdominal multi-organ auto-segmentation using 3Dpatch-based deep convolutional neural network," Sci Rep, vol. 10, no. 1, p. 6204, Dec. 2020, doi: 10.1038/s41598-020-63285-0.

[63] X. Fang and P. Yan, "Multi-organ segmentation over partially labeled datasets with multi-scale feature abstraction," arXiv:2001.00208 [cs], Jun. 2020. Available: http://arxiv.org/abs/2001.00208.

[64] I. J. Goodfellow et al., "Generative adversarial networks," arXiv:1406.2661 [cs, stat], Jun. 2014. Available: http://arxiv.org/abs/1406.2661.

[65] M. Rezaei, H. Yang, and C. Meinel, "Conditional generative refinement adversarial networks for unbalanced medical image semantic segmentation," arXiv:1810.03871 [cs], Oct. 2018. Available: http://arxiv.org/abs/1810.03871.

[66] X. Dong et al., "Automatic multiorgan segmentation in thorax CT images using U-net-GAN," Med. Phys., vol. 46, no. 5, pp. 2157-2168, May 2019, doi: 10.1002/mp.13458.

[67] R. Trullo, C. Petitjean, B. Dubray, and S. Ruan, "Multiorgan segmentation using distance-aware adversarial networks," J. Med. Imag., vol. 6, no. 01, p. 1, Jan. 2019, doi: 10.1117/1.JMI.6.1.014001.

[68] Y. Lei et al., "Male pelvic multi-organ segmentation aided by CBCTbased synthetic MRI," Phys. Med. Biol., vol. 65, no. 3, p. 035013, Feb. 2020, doi: 10.1088/1361-6560/ab63bb.

[69] P.-H. Conze et al., "Abdominal multi-organ segmentation with cascaded convolutional and adversarial deep networks," arXiv:2001.09521 [cs, eess], Jan. 2020. Available: http://arxiv.org/abs/2001.09521.

[70] A. Chakravarty and J. Sivaswamy, "RACE-Net: A recurrent neural network for biomedical image segmentation," IEEE J. Biomed. Health Inform., vol. 23, no. 3, pp. 1151-1162, May 2019, doi: 10.1109/JBHI.2018.2852635.

[71] Q. Yu, L. Xie, Y. Wang, Y. Zhou, E. K. Fishman, and A. L. Yuille, "Recurrent saliency transformation network: Incorporating multi-stage visual cues for small organ segmentation," arXiv:1709.04518 [cs], Apr. 2018. Available: http://arxiv.org/abs/1709.04518.

[72] M. Z. Alom, C. Yakopcic, M. Hasan, T. M. Taha, and V. K. Asari, "Recurrent residual U-Net for medical image segmentation," J. Med. Imag., vol. 6, no. 01, p. 1, Mar. 2019, doi: 10.1117/1.JMI.6.1.014006.
[73] J. Schlemper et al., "Attention gated networks: Learning to leverage salient regions in medical images," arXiv:1808.08114 [cs], Jan. 2019. Available: http://arxiv.org/abs/1808.08114.

[74] A. A. Novikov, D. Major, M. Wimmer, D. Lenis, and K. Buhler, "Deep sequential segmentation of organs in volumetric medical scans," IEEE Trans. Med. Imaging, vol. 38, no. 5, pp. 1207-1215, May 2019, doi: 10.1109/TMI.2018.2881678.

[75] Y. Wang, Y. Zhou, P. Tang, W. Shen, E. K. Fishman, and A. L. Yuille, "Training multi-organ segmentation networks with sample selection by relaxed upper confident bound," arXiv:1804.02595 [cs], Apr. 2018. Available: http://arxiv.org/abs/1804.02595.

[76] V. V. Valindria et al., "Multi-modal learning from unpaired images: application to multi-organ segmentation in CT and MRI," in 2018 IEEE Winter Conference on Applications of Computer Vision (WACV), Lake Tahoe, NV, Mar. 2018, pp. 547-556, doi: 10.1109/WACV.2018.00066.

[77] Y. Zhou et al., "Semi-supervised multi-organ segmentation via deep multi-planar co-training," arXiv:1804.02586 [cs], Nov. 2018. Available: http://arxiv.org/abs/1804.02586.

[78] Y. Zhou et al., "Prior-aware neural network for partially-supervised multi-organ segmentation," arXiv:1904.06346 [cs], Aug. 2019. Available: http://arxiv.org/abs/1904.06346.

[79] Z. Zhang, H. Fu, H. Dai, J. Shen, Y. Pang, and L. Shao, "ET-Net: A generic edge-attention guidance network for medical image segmentation," arXiv:1907.10936 [cs], Jul. 2019. Available: http://arxiv.org/abs/1907.10936.

[80] Y. Zhang, M. T. C. Ying, and D. Z. Chen, "Decompose-and-integrate learning for multi-class segmentation in medical images," arXiv:1906.02901 [cs, eess, stat], Jun. 2019. Available: http://arxiv.org/abs/1906.02901.

[81] A. Sinha and J. Dolz, "Multi-scale self-guided attention for medical image segmentation," arXiv:1906.02849 [cs], Feb. 2020. Available: http://arxiv.org/abs/1906.02849.

[82] (Author Name Not Available), "Segmentation outside the cranial vault challenge," 2015, doi: 10.7303/SYN3193805.

[83] P. F. Raudaschl et al., "Evaluation of segmentation methods on head and neck CT: Auto-segmentation challenge 2015," Med. Phys., vol. 44, no. 5, pp. 2020-2036, May 2017, doi: 10.1002/mp.12197.

[84] J. Yang et al., "Autosegmentation for thoracic radiation treatment planning: A grand challenge at AAPM 2017," Med. Phys., vol. 45, no. 10, pp. 4568-4581, Oct. 2018, doi: 10.1002/mp.13141.

[85] A. L. Simpson et al., "A large annotated medical image dataset for the development and evaluation of segmentation algorithms," arXiv:1902.09063 [cs, eess], Feb. 2019. Available: http://arxiv.org/abs/1902.09063.

[86] A. E. Kavur, M. A. Selver, O. Dicle, M. Barış, and N. S. Gezer, "CHAOS - Combined (CT-MR) healthy abdominal organ segmentation challenge data." Zenodo, Apr. 11, 2019, doi: 10.5281/ZENODO.3362844.

[87] X. Xu, B. Liu, and F. Zhou, "Annotations for body organ localization based on MICCAI LiTS dataset." IEEE DataPort, Jul. 10, 2018, doi: 10.21227/DF8G-PQ27. 